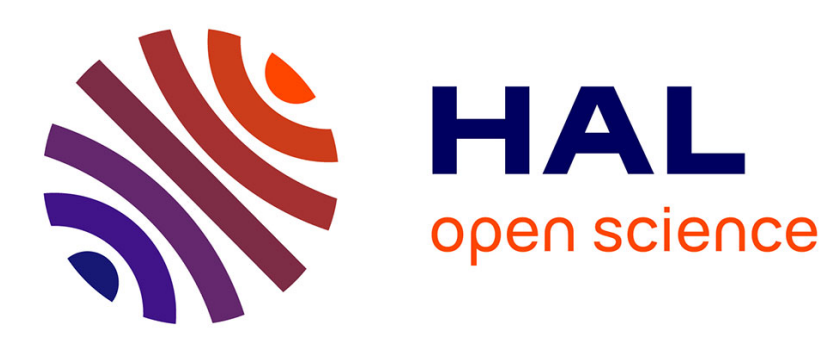

\title{
Pertinence de la comparaison à la loi en d2 en " évaporation " supercritique
}

Stéphane Préau, Roger Prud'Homme, Jalil Ouazzani, Bernard Zappoli

\section{To cite this version:}

Stéphane Préau, Roger Prud'Homme, Jalil Ouazzani, Bernard Zappoli. Pertinence de la comparaison à la loi en d2 en " évaporation " supercritique. Comptes rendus de l'Académie des sciences. Série 2, 2004, 332 (5-6), pp. 397-402. hal-02268402

\section{HAL Id: hal-02268402 \\ https://hal.science/hal-02268402}

Submitted on 27 Nov 2020

HAL is a multi-disciplinary open access archive for the deposit and dissemination of scientific research documents, whether they are published or not. The documents may come from teaching and research institutions in France or abroad, or from public or private research centers.
L'archive ouverte pluridisciplinaire HAL, est destinée au dépôt et à la diffusion de documents scientifiques de niveau recherche, publiés ou non, émanant des établissements d'enseignement et de recherche français ou étrangers, des laboratoires publics ou privés. 


\title{
Pertinence de la comparaison à la loi en $d^{2}$ en « évaporation » supercritique $^{1}$
}

\author{
Stéphane Préau ${ }^{2}$, Roger Prud'homme ${ }^{2}$ Jalil Ouazzani ${ }^{3}$, Bernard Zappoli ${ }^{4}$
}

\begin{abstract}
Résumé
L'évolution temporelle d'une «goutte» en conditions supercritiques dépend de la définition de sa frontière, Dans le cas d'une «goutte» de corps pur s'évaporant dans son gaz, la loi en $d^{2}$ sera ou non satisfaite suivant la position de cette frontière relativement à la zone de fort gradient en densité. Le suivi de la loi en $d^{2}$ n'apparaît alors pas comme un critère pertinent pour caractériser les régimes de évaporation» quasi-stationnaires. Le cas de la «goutte » d'oxygène s' « évaporant » dans une atmosphère d'hydrogène est ensuite discuté.
\end{abstract}

\begin{abstract}
Relevance of the comparison to the $d^{2}$ law in supercritical 'vaporization'. The temporal evolution of a 'droplet' in supercritical conditions depends on the definition of its boundary. In the case of a pure 'droplet' 'vaporizing' in its gas, the $d^{2}$ law is, or is not, satisfied depending on the location of the boundary in relation to the strong density gradient area. Consequently, the $d^{2}$ law is not a relevant criterion of comparison in order to characterize the quasi-steady 'vaporization' process. The case of an oxygen 'droplet' 'vaporizing' in hydrogen is then discussed.
\end{abstract}

Mots-clés: Transferts thermiques; Évaporation; Quasi-stationnaire; Supercritique

Keywords: Heat transfer; Vaporization; Quasi-steady; Supercritical

\section{Abridged English version}

The vaporization process of a droplet a the boiling temperature can be studied by use of the quasi-steady analysis provided that the characteristic diffusion time in the gas phase js much smaller than the droplet lifetime. Unsteady terms in the balance equations are then neglected and it is shown that the squared droplet diameter varies linearly in time: this is the $d^{2}$ law. The comparison of the squared droplet radius evolution to the $d^{2}$ law is often considered as a criterion to assess the nature of the vaporization process. However in supercritical conditions, the definition of the 'droplet' boundary remains arbitrary and the squared droplet radius evolution depends on the boundary definition.

In the case of a slightly supercritical pure 'droplet' vaporizing in a much hotter atmosphere of its gas, our numerical simulations show that the $d^{2}$ law is only satisfied when the 'droplet' is bounded by an isodensity located at the end of the strong gradient that follows the steady area. When the 'droplet' boundary is located outside this strong density gradient, the 'droplet' expands in the initial period. If the droplet' boundary is located in the strong density gradient, the squared 'droplet' radius evolves below the linear law. Moreover, in the case of the 'droplet' evolution that follows the $d^{2}$ law, the mass flow rate evolution at the 'droplet' boundary does not correspond to the quasi-steady prediction.

A quasi-steady analysis adapted to slightly supercritical configurations [7] can predict the evolution of a 'droplet' bounded by an isodensity located in the strong density gradient area: the $d^{2}$ law is no longer a characteristic criterion of quasi-steadiness.

In the case of a pure oxygen droplet' vaporizing in an atmosphere of hydrogen, the 'droplet' boundary is either chosen at the critical isothermal or at the critical mass fraction

\footnotetext{
${ }^{1}$ C.R. Mecanique 332 (2004) 397-402. Disponible sur Internet le 24 avril 2004.

${ }^{2}$ Laboratoire de modélisation en mécanique, 4, place Jussieu, case 162, 75252 Paris cedex 05, France

${ }^{3}$ Arcofluid, Les Bureaux de I 'Arche, 5, rue des allumettes 13086 Aix en Provence cedex 02, France

${ }^{4}$ CNES, 18. avenue Edouard Belin, 31055 Toulouse cedex, France
} 
location: the density is not a priori constant at the droplet' boundary. However, according to the numerical simulations of [9], a constant density value is reached very rapidly at the 'droplet' boundary and the results obtained in the case of the pure 'droplet' vaporizing in its gas could apply qualitatively.

\section{Introduction}

Le problème de l'évaporation d'une goutte de température uniformément égale à la température de vapeur saturante dans une atmosphère plus chaude et isobare peut être traité analytiquement par l'analyse de type Quasi- Stationnaire (QS) développée par Godsave [1] et Spalding [2]. Une hypothèse doit pour cela être effectuée : le temps caractéristique de diffusion en phase gazeuse est supposé très inférieur au temps de vie de la goutte. Ceci se vérifie en pratique lorsque $\varepsilon=\rho_{\infty}^{\prime} / \rho_{L}^{\prime}<<1$ avec $\rho_{\infty}^{\prime}$ la densité de l'atmosphère initiale et $\rho_{L}^{\prime}$ celle du liquide. L'intégration en phase gazeuse des équations de bilan de masse et d'énergie où les termes instationnaires sont négligés au premier ordre conduit alors à la détermination du temps de vie de la goutte et à l'établissement de la loi en $d^{2}$ : le carré du diamètre de la goutte varie linéairement avec le temps. D'autre part, cette analyse montre que les profils en température et en densité sont stationnaires dans un repère où le rapport

$r^{\prime} / r_{\mathrm{g}}{ }^{\prime}\left(t^{\prime}\right)$ (avec $r^{\prime}$ la coordonnée radiale, $t^{\prime}$ le temps et $r_{\mathrm{g}}{ }^{\prime}\left(t^{\prime}\right)$ le rayon de la goutte) est choisi comme variable. Si le paramètre $\varepsilon$ n'est plus très inférieur à un, la théorie instationnaire développée par Crespo et Liñian [3] décrit le processus. La loi en $d^{2}$ n'est en particulier plus vérifiée.

C'est le critère du respect à la loi en $d^{2}$ qui est généralement invoqué dans les études expérimentales ou numériques pour juger de la validité de l'hypothèse QS.

Aux pressions supercritiques. il n'y a plus d'interface liquide-gaz, l'ensemble du domaine est en effet monophasique, et le choix d'une frontière de référence délimitant la «goutte » supercritique ne peut être qu'arbitraire. Dans le cas de l' «évaporation» d'une "goutte» d'oxygène dans une atmosphère d'hydrogène, cette frontière est ainsi définie par l'isotherme critique de mélange à la pression considérée chez Lafon [4] et Yang et al. [5] ou par le lieu où la fraction massique égale la fraction massique critique à la pression considérée chez Haldenwang et al. [6]. L'importance de la définition de la frontière de référence est soulignée dans ce dernier article : à une pression de 100 bars, la «goutte» délimitée par la composition critique se dilate en début du processus, ce qui n'est pas le cas de la «goutte» délimitée par l'isotherme critique. L'évaluation de la pertinence de l'hypothèse QS par la comparaison avec la loi en $d^{2}$ apparaît déjà délicate : pour une même configuration, telle définition de la « goutte » supercritique conduira à réfuter la validité de l'hypothèse QS, telle autre la confirmera.

Nous étudions dans ce qui suit l'influence du choix de la frontière délimitant une «goutte» supercritique de corps pur «s'évaporant» dans son gaz sur la forme du profil de récession du carré de son rayon en nous appuyant sur les résultats présentés dans [7]. Le cas de l'«évaporation » d'une «goutte» d'oxygène dans une atmosphère d'hydrogène est ensuite discuté à la lumière des résultats obtenus dans le cas du corps pur.

\section{Structure spatiale de l' «évaporation » supercritique}

A une pression supercritique $P^{\prime}$, telle que $P^{\prime} / P^{\prime}{ }_{c}=1,00004$ avec $P_{c}^{\prime}$, la pression critique, considérons une poche de fluide pur à la densité critique $\rho_{c}^{\prime}$ soudainement injectée dans une 
atmosphère infinie plus chaude de ce même fluide, telle que $\varepsilon=\rho_{\infty}^{\prime} / \rho_{c}^{\prime}=0,025 \ll 1$. Les conditions initiales sont donc de type créneau en densité.

Passé l'instant initial, trois zones peuvent être distinguées sur les profils en densité :

- Une zone centrale stationnaire $D_{o}$ délimitée asymptotiquement par une isodensité $\rho_{0}^{\prime}$ définissant un domaine de rayon $r^{\prime}{ }_{\mathrm{o}}\left(t^{\prime}\right)$ avec $t$, le temps;

- Une zone de fort gradient en densité comprise entre les isodensités $\rho_{o}^{\prime}$ et $\rho_{m}^{\prime}$, où, d'après l'analyse asymptotique menée en [7], $\rho_{m}^{\prime}$ est telle que

(1)

$$
\frac{t_{\mathrm{dif}}^{\prime}\left(\rho_{m}^{\prime}\right)}{t_{\mathrm{vie} D_{o}}^{\prime}}=\mathrm{O}(1)
$$

avec $t_{\text {dif }}^{\prime}\left(\rho_{m}^{\prime}\right)$, le temps caractéristique de diffusion en $\rho_{m}^{\prime}$ et $t^{\prime}{ }_{\text {vieDo }}$ le temps de vie du domaine $D_{\text {o. }}$. Il est par ailleurs montré dans [7] que $\rho_{m}^{\prime}$ se situe dans la zone où le débit surfacique $\rho^{\prime} v^{\prime}$ est maximal. Notons $D_{m}$ le domaine délimité par l'isodensité $\rho_{m}^{\prime}$ et $r_{m}^{\prime}\left(t^{\prime}\right)$ son rayon;

- Une zone extérieure suit enfin la zone de fort gradient en densité. Définissons un dernier domaine, $D_{n}$ rayon $r_{n}^{\prime}\left(t^{\prime}\right)$, délimité par une isodensité $\rho_{n}^{\prime}$ située dans cette zone extérieure à la zone de fort gradient en densité et donc telle que: $\rho_{n}^{\prime}<\rho_{m}^{\prime}$.

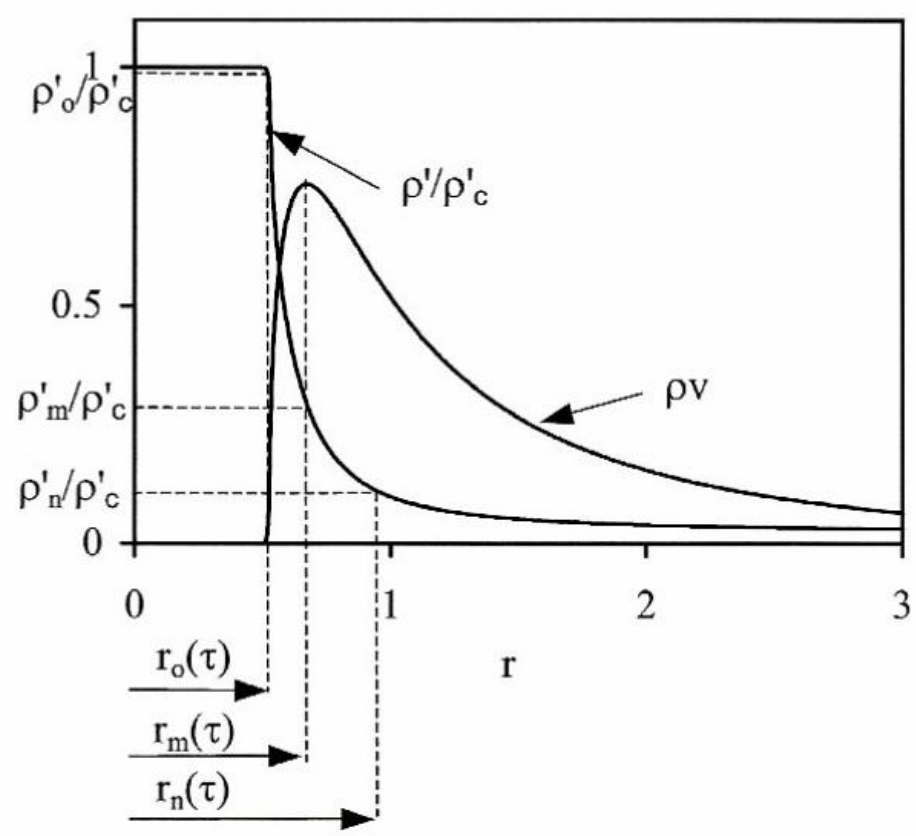

Fig. 1. Profils numériques en densité et en débit surfacique adimensionnel à un instant donné. Les rayons des domaines $D_{0}, D_{m}$, et $D_{n}$, sont situés sur ces profils. Le débit surfacique de référence pour l'adimensionalisation est choisi égal à $\rho_{\infty}^{\prime} r_{\text {in }}^{\prime} / t^{\prime}{ }_{d i f}\left(\rho_{\infty}^{\prime}\right)$

Fig. 2 .Numerical profiles in density and dimensional unit mass flow rate at a given time. The radius of the domains $D_{0}, D_{m}$, and $D_{n}$, are situated on the profiles. The reference unit mass flow rate for scaling has been chosen equal $\rho_{\infty}^{\prime} r_{i n}^{\prime} / t_{d i f}^{\prime}\left(\rho_{\infty}^{\prime}\right)$

Les rayons adimensionnels ( $r=r^{\prime} / r^{\prime}$ in avec $r_{\text {in }}^{\prime}$ le rayon initial de la poche de fluide dense) de ces trois domaines sont représentés sur la Fig. 1. 


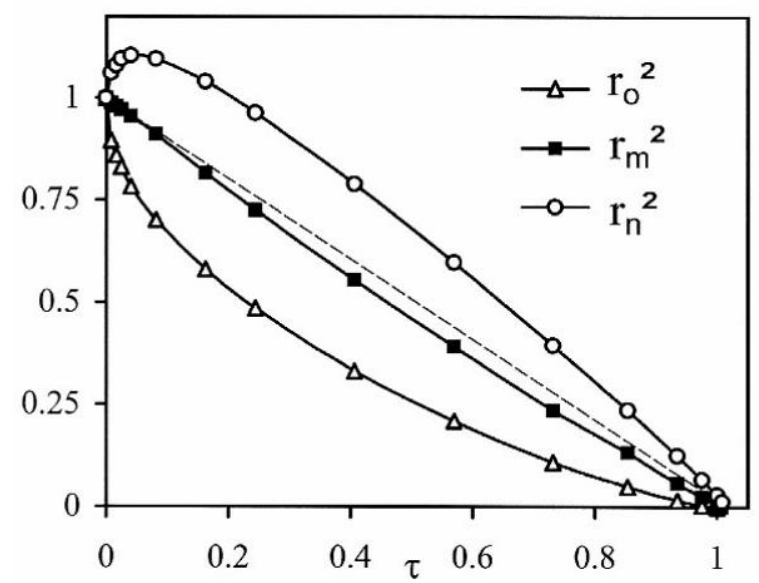

Fig. 2. Évolution temporelle de $r_{0}^{2}(\tau), r_{m}^{2}(\tau)$ et $r_{n}^{2}(\tau)$

Fig. 2. Time evolution of $r_{0}^{2}(\tau), r_{m}^{2}(\tau)$ and $r_{n}^{2}(\tau)$

L'évolution des carrés des rayons de ces trois domaines est tracée sur la Fig. 2 en fonction du temps adimensionnel $\tau=t^{\prime} / t_{\text {vieDo }}^{\prime}$ Ce graphe est obtenu par résolution numérique directe des équations de Navier- Stokes discrétisées par une méthode de type volumes finis. Il ressort de ce graphe que la loi en $d^{2}$ n'est vérifiée que dans le cas du domaine $D_{m}$, de rayon $r_{m}(\tau)$, Par ailleurs, seul le domaine $D_{n}$ se dilate en début de processus. De façon plus générale, les domaines subissant une dilatation initiale sont délimités par des isodensités $\rho$ ' telles que

$\rho^{\prime}<\rho_{m}^{\prime}$, (isodensités situées dans la zone extérieure à la zone de fort gradient en densité). Les domaines régressant au contraire plus rapidement que le domaine $D_{m}$ aux temps courts sont, eux, délimités par des isodensités $\rho$ ' situées dans la zone de fort gradient et qui vérifient à ce titre : $\rho_{m}^{\prime} \leq \rho^{\prime} \leq \rho_{o}^{\prime}$.

Notons que le temps de vie de la «goutte» dépend de la définition de sa frontière: les temps de vie des domaines délimités par les isodensités situées dans la zone de fort gradient peuvent être considérés égaux au premier ordre (les temps de vie des domaines $D_{o}$ et $D_{m}$ diffèrent de $1 \%$ en valeur relative dans la configuration testée). Les temps de vie des domaines délimités par des isodensités situées hors de la zone de fort gradient sont, eux, d'autant plus élevés que la densité $\rho_{n}^{\prime}$ est choisie petite.

\section{Pertinence de la comparaison à la loi en $d^{2}$ aux pressions proches de la pression critique}

Des relevés expérimentaux qui suivraient la frontière de la «goutte» en $r_{o}^{\prime}\left(t^{\prime}\right)$ (la frontière du domaine $D_{o}$ est localisée juste au début de la zone où le gradient en densité est maximal, zone justement capturée par les mesures optiques [8] inciteraient à conclure sur l'instationnarité du phénomène, la loi en $d^{2}$ n'étant pas satisfaite. Un suivi numérique du domaine de rayon $r_{n}^{\prime}\left(t^{\prime}\right)$ la «goutte» s'identifiant alors au domaine $D_{n}$, conduirait aux mêmes conclusions. Le suivi du domaine de rayon $r_{m}^{\prime}\left(t^{\prime}\right)$ qui vérifie la loi en $d^{2}$ plaiderait au contraire en faveur de la pertinence de l'analyse QS. Cette dernière conclusion serait cependant assez hâtive : comme indiqué sur la Fig. 3, le débit adimensionnel en $r_{m}^{\prime}\left(t^{\prime}\right)$ ne correspond pas à celui prédit par la théorie QS telle que présentée dans $[1,2]$ pour des configurations nettement subcritiques, que nous nommerons par la suite analyse QS « classique». La divergence observée pour les temps proches de zéro s'explique par le très fort 
gradient en densité lié à la discontinuité des conditions initiales. Cette divergence est cependant probablement bloquée aux temps très courts par des effets acoustiques.

Une analyse de type QS spécifiquement adaptée aux pressions légèrement sub ou super-critiques [7] retrouve sous forme analytique les lois de régression des rayons $r_{o}^{\prime}\left(t^{\prime}\right)$ et $r_{m}^{\prime}\left(t^{\prime}\right)$ présentées sur la Fig. 2 ainsi que l'évolution temporelle du débit en $r_{m}^{\prime}\left(t^{\prime}\right)$ présentée sur la Fig. 3. Pour le domaine $D_{o}$ de rayon $r_{o}^{\prime}\left(t^{\prime}\right)$ la loi en $d^{2}$ est asymptotiquement retrouvée lorsque la pression devient nettement subcritique.

La comparaison à la loi en $d^{2}$ s'avère donc peu fiable pour caractériser le régime d'《évaporation» dans la configuration décrite ici puisque des lois de régressions du carré du rayon de la «goutte» non linéaires dans le temps peuvent être retrouvées par une analyse de type QS spécifique, à condition toutefois que la frontière de la «goutte» soit située dans la zone de fort gradient en densité.

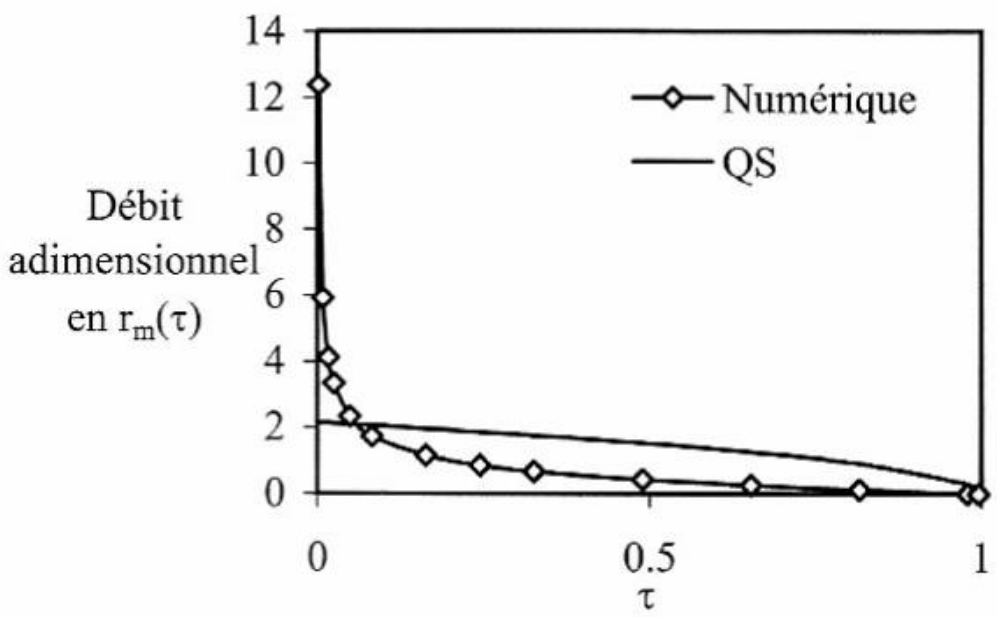

Fig. 3. Débit adimensionnel en $r_{m}(\tau)$ : évolution numérique et calculée par la théorie QS [1,2].

Fig. 3. Adimensional mass flow rate in $r_{m}(\tau)$ : numerical evolution. and calculated by the QS theory $[1,2]$.

\section{4. «Évaporation » d'une «goutte» d'oxygène dans une atmosphère d'hydrogène}

Dans le cas de l' évaporation» supercritique d'une «goutte» de corps pur dans un mélange gazeux, la frontière de la «goutte» est comme nous l'avons dit, le plus souvent choisie sur l'isotherme critique de mélange $T_{c}^{\prime}\left(P^{\prime}\right)$ ou définie à l'endroit où la fraction massique atteint la valeur critique $Y_{c}\left(P^{\prime}\right)$. Or, pour un mélange, à pression fixée, la loi d'état du gaz est de la forme : $\rho^{\prime}=f\left(T^{\prime}, \mathrm{Y}\right)$ et à une température ou une composition données correspond une infinité de valeurs pour la densité. La densité à la frontière $T_{c}^{\prime}\left(P^{\prime}\right)$ ou $Y_{c}\left(P^{\prime}\right)$ varie donc a priori dans le temps. Les simulations numériques menées par Harstad et Bellan [9] dans le cas d'une «goutte» d'oxygène s' «évaporant» dans une atmosphère d'hydrogène, montrent que des relations du type $\rho^{\prime}=g\left(T^{\prime}\right)$ et $\rho^{\prime}=h(Y)$ sont rapidement atteintes dans le domaine, passé l'instant initial. En d'autres termes, et quelque soit la définition de la «goutte», la densité à sa frontière atteint rapidement une valeur quasi-constante. Un parallèle avec le cas de la « goutte» de corps pur s' " évaporant» dans son gaz pourrait dès lors être envisagé : une dilatation initiale de la « goutte» d'oxygène indiquerait que la densité à la frontière de cette dernière se situe hors de la zone de fort gradient. Des profils de récession du carré du rayon de la «goutte» passant sous la courbe linéaire indiqueraient plutôt que la densité à la frontière est localisée dans la zone de fort gradient en densité.

Les résultats présentés dans [4] et [5] pour des pressions supercritiques de l'ordre de $100 \mathrm{~atm}$ montrent que la loi en $d^{2}$ n'est pas satisfaite, le carré du rayon de la «goutte» d'oxygène évoluant sous la courbe linéaire. En poursuivant l'analogie avec le cas du corps pur, ceci témoignerait alors non pas 
du caractère instationnaire du phénomène mais plutôt du fait qu'à de telles pressions, la densité atteinte à la frontière de la « goutte» se situe dans la zone de fort gradient, un comportement de type QS ne pouvant alors être écarté.

\section{Conclusions}

Compte tenu des résultats obtenus dans le cas d'une «goutte» de corps pur s' «évaporant» dans son gaz à des pressions proches de la pression critique, le critère de la correspondance à la loi en $d^{2}$ pour juger de la nature du régime d' «évaporation»d'une «goutte» de corps pur dans un mélange à hautes pressions devrait être considéré avec précautions.

\section{Références}

[1] G.A.E. Godsave, The burning of single drops of fuel, in: Fourth Symposium on Combustion, 1953, p. 818.

[2] DB. Spalding, The combustion of liquid fuels, in: Fourth Symposium on Combustion, 1953, p. 854.

[3] A. Crespo, A. Liñan, Unsteady effects in droplet evaporation and combustion. Combust. Sci. Technol. 11(1975) 9.

[4] P. Lafon, Modélisation et simulation numérique de l'évaporation et de la combustion de gouttes à hautes pressions, Thèse ONERA-Paris VI. 1994.

[5] V. Yang. N,N. Lin. I.S. Shuen. Vaporization of liquid oxygen (LOX) droplets at supercritical conditions. 30th Aerospace Sciences Meeting. AIAA paper 92-0103, 1992.

[6] P. Haldenwang C. Nicoli, J. Daou. High pressure vaporization of LOX droplet crossing the critical conditions, Int. J. Heat Mass. Transfer 39 (1996) 3453.

[7] S. Préau. Étude théorique et numérique de 1 «évaporation» d'une «goutte» de densité critique le long d'une isobare légèrement supercritique. Thèse de l'université Paris 6, 2003.

[8] K. Harstad, J, Bellan, An all-pressure fluid drop model applied to a binary mixture: heptane in nitrogen, Int. J. Multiphase Flow 26 (2000) 1675.

[9] K. Harstad, J. Bellan, Isolated fluid oxygen drop behavior in fluid hydrogen at rocket chamber pressures, Int. J. Heat Mass Transfer 41 (1998) 3537. 